\title{
Work of prospective on the adaptation of the viticulture to climate change: Which series of events could support various adaptation strategies? / Travaux de prospective sur l'adaptation de la viticulture au changement climatique : quelles séries d'événements pourraient favoriser différentes stratégies d'adaptation?
}

Patrick Aigrain $^{1}$, Françoise Brugière ${ }^{1}$, Eric Duchêne ${ }^{2}$, Inaki Garcia de Cortazar-Atauri ${ }^{3}$, Jacques Gautier ${ }^{4}$, Eric Giraud-Héraud ${ }^{5}$, Hervé Hannin ${ }^{6}$, Nathalie Ollat ${ }^{5}$, et Jean-Marc Touzard ${ }^{7}$

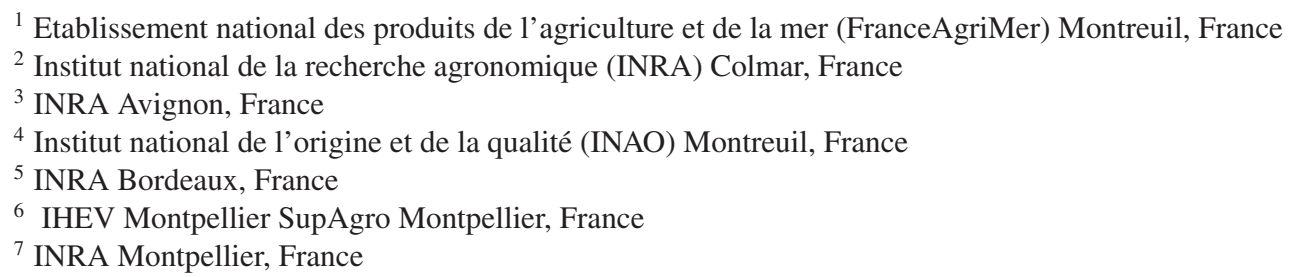

\begin{abstract}
A multidisciplinary group of researchers and experts of public bodies working on the French wine sector met together to elaborate a foresight exercice with the construction of the plausible ways leading to four different adaptation strategies to climate change of the French wine sector to climate change.

For the implementation of this work, the group, considered a single climatic scenario and

- difined four strategies of adaptation incorporating both geographical mobility and of integration of technological changes,

- used assumptions from three sources: assumptions from researchers of various disciplines in their own disciplinary field, pre-existing assumptions from former foresight works and assumptions defined according to the statements of actors of the sector on the impacts of climate change and on the possible methods of adaptation. All these assumptions were written in the form of simple sentences, giving one version and its contrary.

The various assumptions were selected and then combined between them in order to create a blend within the disciplinary fields, without however inferring of orientation.

The result of this work is the join-construction of four plausible ways each one leading to one of the four preselected strategies of adaptation: "conservative" strategy where the evolutions are limited, a "nomade" strategy where the relocalisation of the vineyards is the main vector of adaptation, an "innovating" strategy where the adaptation is the result of the systematic integration of innovations, and finally towards a "liberal" strategy largely opened to any kind of changes.

These ways and strategies are not supposed to give a predictive image of the future. However while proposing possible or plausible futures, this work provides material which could be debated within the sector, without immediate challenges that could possibly bias the discussion

From the expression of the challenges and consequences of the various ways and strategies in which they result, the actors of the French wine sector will be able to start a strategic reflection.

The presentation will be focused on the description of the four ways leading to adaptation strategies and their elaboration.

Methodology is specified in another article "Adaptation to the climate change: interest of a prospective approach", same authors.
\end{abstract}

\begin{abstract}
A l'horizon 2050, les vignobles français et leurs filières vont devoir s'adapter au changement climatique, qui modifiera à la fois les conditions de production des vins, leurs caractéristiques organoleptiques et leurs marchés. A cette échéance, d'autres facteurs tels que les évolutions des préoccupations de santé et d'environnement, les changements géopolitiques ou technologiques, les nouvelles
\end{abstract}

conditions de consommation du vin ou encore les modifications de politique agricole affecteront le secteur vitivinicole.

Comment les acteurs de la vigne et du vin pourront-ils s'adapter à un contexte où le changement climatique va se combiner à des changements socio-économiques souvent incertains ? Les leviers d'adaptation envisageables sont 
multiples : nouveaux cépages, modifications des pratiques viticoles et œnologiques, stratégies de localisation des producteurs et négociants, réorganisation de la filière et de son cadre réglementaire, redéfinition des politiques de recherche et d'innovation, ... De telles actions sont déjà mises en œuvre ou envisagées, mais souvent sans vision très claire, sans réflexion collective sur des futurs possibles affectés par le changement climatique. Réfléchir et imaginer ces futurs possibles devient stratégique pour les acteurs de la vigne et du vin et pour la Recherche censée les accompagner.

Pour étudier les impacts du changement climatique et les adaptations potentielles du secteur viti-vinicole français, l'INRA a soutenu entre 2012 et 2016 le projet LACCAVE, au sein d'un programme plus large sur l'Adaptation au Changement Climatique de l'Agriculture et de la Forêt (métaprogramme ACCAF). Ce projet a associé des chercheurs de plusieurs disciplines (climatologie, génétique, agronomie, œnologie, économie, sociologie, ... ), issus de vingt-trois équipes de l'INRA, du CNRS, d'universités et d'écoles d'ingénieurs ${ }^{1}$.

Une des opérations phare du projet LACCAVE a été la conduite d'un exercice de prospective. Ses objectifs étaient :

- Identifier et explorer différentes stratégies d'adaptation pour la viticulture française dans un contexte de changement climatique, à l'horizon 2050 ;

- Tester une méthode pour élaborer des 《 chemins » favorisant la mise en œuvre de ces stratégies d'adaptation ${ }^{2}$;

- Développer une vision commune et des pratiques de travail en réseau entre chercheurs et organisations de la filière.

\section{Une méthode originale de prospective}

Un groupe multi-disciplinaire de chercheurs et d'experts d'organismes publics de l'accompagnement de la filière viti-vinicole française (INAO et FranceAgriMer) s'est réuni pour travailler en prospective à la co-construction des chemins plausibles menant à quatre stratégies différentes d'adaptation de la filière viticole française au changement climatique.

Pour la réalisation de ses travaux, le groupe, à partir d'un scénario climatique prédéfini,

- a fixé quatre stratégies d'adaptation articulant plus ou moins de mobilité géographique et d'intégration d'évolutions technologiques,

- a accumulé un stock d'hypothèses à partir des trois sources suivantes : les hypothèses des chercheurs de différentes disciplines sur leur propre champ disciplinaire, les hypothèses préexistantes dans des travaux

\footnotetext{
${ }^{1}$ Equipes de recherche des centres INRA de Bordeaux, Montpellier, Avignon, Colmar, Angers, Paris, du CNRS Rennes, de Bordeaux Sciences Agro, de Montpellier SupAgro, des Universités de Limoges, Dijon, Bordeaux.

${ }^{2}$ L'objectif de ce travail est l'exploration de voies d'adaptation aux changements climatiques et ne prend pas en compte les possibilités d'atténuation de la contribution du secteur Vignes et Vins à ces changements climatiques, considérant ici implicitement que ces changements sont largement déterminés par des facteurs extra sectoriels.
}

antérieurs de prospective et des hypothèses rédigées à partir des discours des acteurs de la filière sur les impacts du changement climatique et les modalités d'adaptation possibles. Toutes ces hypothèses sont rédigées sous la forme de phrases simples, exprimées en recto et en verso. Exemple d'hypothèse : La consommation de vin augmente Vs La consommation de vin diminue.

Les différentes hypothèses ont été sélectionnées puis combinées entre elles de façon à faire interférer les champs disciplinaires, sans toutefois inférer d'orientation.

Le résultat de ce travail est la construction de quatre chemins plausibles conduisant chacun à une des quatre stratégies d'adaptation présélectionnées : vers une stratégie 《conservatrice » où les évolution sont limitées, vers une stratégie 《nomade » où le déplacement des vignobles est le vecteur d'adaptation privilégié, vers une stratégie « innovante » où l'adaptation se fait par l'intégration systématique des innovations, et enfin vers une stratégie « libérale » largement ouverte aux changements de toutes natures.

Les chemins ont été décrits en organisant un récit à partir des seize micro-scénarios et d'un canevas commun de quatre familles de questions :

- le contexte général du système vignes et vins avec en particulier la politique de santé publique vis-à-vis de l'alcool, les objectifs du financement de la recherche par l'Etat, l'existence ou non d'une gestion publique de l'espace agricole ;

- le contexte viti-vinicole international et plus particulièrement l'évolution de la définition internationale $\mathrm{du}$ vin, les règles internationales d'étiquetage de provenance des vins, la nature et l'évolution de l'intervention publique européenne sur le vignoble (y compris les plantations) ;

- le contexte viti-vinicole local \& national avec les relations entre la filière et la recherche, les échelles de compréhension des impacts du changement climatique sur la vigne, la capacité structurelle d'adaptation des exploitations, l'attitude des consommateurs face à l'évolution du goût des vins, ou l'évolution de la demande en vins biologiques (en lien avec la dimension internationale) ;

- les conséquences sur la filière, incluant sa gouvernance (à l'échelle régionale notamment), le poids relatif des régions viticoles, l'évolution de ses performances économique notamment à l'export, le poids des AOP dans les échanges vinicoles.

Chacun des chemins correspond à une combinatoire unique de réponses apportées à ces familles communes de questions.

\section{Les chemins vers les stratégies d'adaptation}

\subsection{Le chemin vers la stratégie conservatrice}

\section{Résumé}

Dans un contexte de pression des autorités sanitaires sur les boissons contenant de l'alcool et d'une gestion des terres et de l'eau en priorité destinées aux cultures alimentaires, la filière, peu liée avec la recherche, 
perçoit le changement climatique comme une menace. Sur la base d'un contenu culturel et paysager mis en avant pour bien différencier le vin d'une boisson seulement alcoolisée, les IG et les régions qui les produisent constituent les îlots de résistance d'une viticulture qui se rétracte.

Cf. tableau 1.

\subsection{Des politiques publiques défavorables à la filière vignes et vins}

La filière viticole n'est pas parvenue à proposer des orientations claires sur les pistes de recherche à explorer pour faciliter l'adaptation au changement climatique. Cela a facilité un désengagement de l'Etat dans le financement de la recherche sur la vigne et le vin.

La constitution de bases de données à l'échelle des parcelles s'est essoufflée faute de moyens et de mise en évidence de leur intérêt opérationnel par des utilisations pertinentes. La compréhension des effets de la variabilité du climat sur la phénologie de la vigne ou sur la répartition spatiale et temporelle des ravageurs n'a pas vraiment progressé. Les producteurs dont les compétences et la formation se sont maintenues, se trouvent confrontés aux aléas climatiques de plus en plus fréquents avec en moyenne une récolte « normale » sur quatre, sans pouvoir s'en protéger. Dans ce contexte le changement climatique est perçu globalement comme une menace. La production en bio, qui demande à la fois une accumulation d'observations et d'expériences et une forte technicité, n'est pas facilitée.

Les ressources en eau et en terre fertile sont préférentiellement destinées aux cultures alimentaires, notamment les plaines irrigables du Sud de la France où tout renouvèlement du vignoble est soumis à autorisation. Les producteurs qui ont une réserve foncière sur leur exploitation ou à proximité, se tournent vers des parcelles plus en altitude, moins bien exposées ou bénéficiant d'une réserve utile en eau plus importante. Les vignobles sont strictement circonscrits à leurs territoires historiques.

\section{Sans alternative innovante, la filière se sécurise dans les traditions...}

Dans ce contexte où volume et qualité de production tendent à être plus aléatoires, les opérateurs en aval du marché proposent des contrats aux producteurs afin de sécuriser leurs approvisionnements.

L'absence d'orientation stratégique et de moyens de recherche consacrés à la filière se fait sentir également en œnologie. Les pratiques correctives qui auraient permis de maintenir le profil gustatif et aromatique des vins malgré l'évolution des conditions de maturation des raisins n'ont pas été développées à grande échelle. Les technologies de désalcoolisation restent hors de portée de la majorité des producteurs. Sans pression d'innovation, la définition internationale du vin et la panoplie des pratiques œnologiques autorisées restent restrictives.

De son coté l'Organisation Mondiale de la Santé (OMS) renforce sa lutte contre les maladies non épidémiques et les boissons alcoolisées sont particulièrement ciblées. L'organisation fait tout son possible pour limiter la distribution des vins. La règle de 《l'entièrement obtenu $»^{3}$ est finalement adoptée à l'Organisation Mondiale des Douanes car jugée défavorable au développement des échanges vitivinicoles, ce qui est cohérent avec le ban de l'OMS sur l'alcool. Les assembleurs internationaux restent marginaux. Le recul de la demande globale des pays importateurs conduit à maintenir un contrôle administratif des plantations et un niveau minimal d'interventions très éco-conditionnelles.

Néanmoins, la part de marché des vins français à l'export se maintient voire se développe du fait du poids des A.O. (considérées en partie comme des biens culturels et moins sensibles aux taxes à l'importation dorénavant autorisées sur les boissons alcooliques) et les régions productrices de vins à indication géographique protégée (IGP) ou sans indication géographique (sans IG) connaissent un recul important.

\section{...pour un cercle restreint de consommateurs}

Le vin conserve suffisamment de consommateurs qui mettent en avant l'hédonisme et la convivialité. La notion de terroir, l'enracinement géographique, la stabilité des pratiques, font partie des histoires que ces consommateurs aiment à se raconter. Le support narratif de la production permet de justifier l'évolution des profils aromatiques, rend acceptable certains défauts causés par les nouvelles conditions climatiques et permet des expérimentations individuelles. Rare et aléatoire, le vin devient cher et permet la rentabilité d'îlots de traditions mâtinées d'expérimentation.

Sur de nouveaux territoires désormais propices à la culture de la vigne, quelques amateurs expérimentent de nouveaux vins locaux et des investisseurs font étudier les possibilités d'y reproduire le modèle de création de valeur déjà en vigueur dans ce qui reste des vignobles traditionnels.

\subsection{Le chemin vers la stratégie innovante}

Résumé
Les enjeux environnementaux et sanitaires sont un moteur
d'une évolution qui conduit à l'intégration d'un nombre
croissant d'innovations de la vigne à la cave. Cette
évolution est autorisée dans un contexte favorable à une
certaine stabilité des régions viticoles françaises et au
développement d'une politique contraignante en matière
de zonage agricole dans l'UE et relativement libérale en
matière de conditions d'élaboration des vins.
Cf. Tableau 2.

Le contexte économique général est favorable à une certaine stabilité des régions viticoles françaises en termes de surface et de volume. Au plan mondial un ban de l'OMS sur les boissons alcoolisées dont le vin fait partie - et des règles douanières contraignantes visent à limiter la consommation de vin en freinant les échanges commerciaux internationaux et en entravant l'implantation d'assembleurs internationaux près des centres de consommation. L'UE souhaite

\footnotetext{
3 Exigence d'une même provenance douanière non préférentielle des raisins et des vins qui en sont issus, pour pouvoir étiqueter l'origine douanière d'un vin.
} 
Tableau 1.

\section{Chemin conduisant préférentiellement à une stratégie d'adaptation conservatrice}

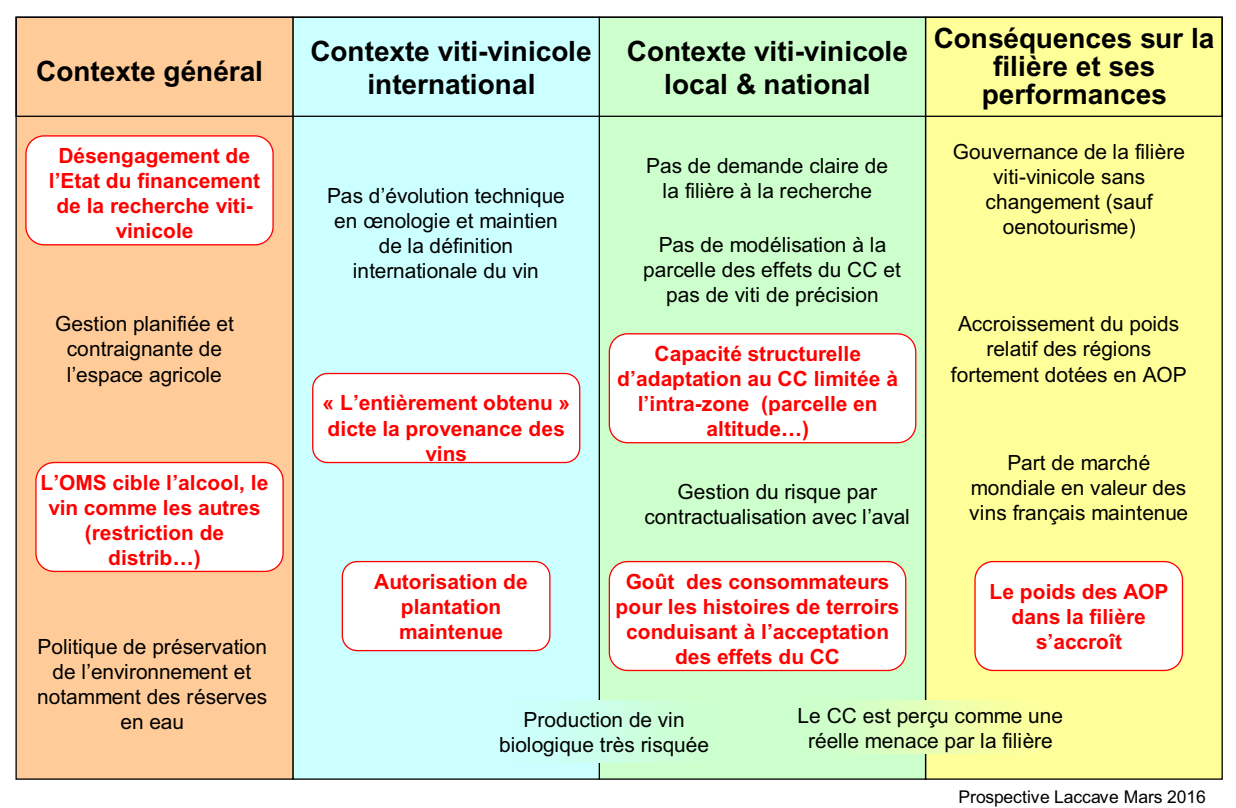

répondre à ces contraintes en libéralisant, sans aides publiques, les pratiques des assemblages intra-UE de Vins sans Indication Géographique (VSIG) issus de cépages sans autorisation nationale; elle espère ainsi augmenter le potentiel de production des vins « entièrement obtenus » dans les zones à IG et permettre l'émergence de marques intra-UE de vins sans IG. Des opérateurs européens améliorent de la sorte leur compétitivité sur les segments d'entrée de gamme et leur positionnement sur le marché intérieur UE au détriment des pays tiers.

En Europe les attentes sociétales associent de plus en plus à l'enjeu climatique, la préservation des ressources naturelles (eau, biodiversité), la protection de l'environnement et des citoyens et la sécurité alimentaire. Par conséquent, des choix politiques tranchés se traduisent par une planification poussée de l'espace agricole, qui réserve les terres les plus fertiles aux cultures vivrières, et limite la culture de la vigne aux zones de «moindre fertilité agronomique », en dépit d'une libéralisation affichée des plantations.

Innovations technologiques pour s'adapter à des zones et des conditions plus contraignantes

Cette politique favorise le maintien de la viticulture dans les zones traditionnelles ou à leur périphérie. Elle s'accompagne d'un soutien à la R\&D publique et privée pour la mise au point d'innovations techniques qui permettent de relever ces défis. Notamment, l'intégration de plus en plus poussée des techniques associées à la viticulture de précision favorise le développement d'une ingénierie agro-climatique fondée sur des connaissances très fines, ce qui permet de mieux tirer parti du territoire et de ses contraintes climatiques et pédologiques. Cette dynamique des connaissances agro-climatiques permet aussi le développement de démarches agro-écologiques, d'une viticulture biologique avec plusieurs évolutions possibles. Ces viticultures peuvent se développer du fait de meilleures connaissance et gestion des risques, qui résultent certes de l'intégration de nouveaux savoirs, mais aussi de dispositifs d'assurances et de contrats.

Sur les territoires viticoles, les conflits possibles entre poursuite du développement viticole et préservation des ressources naturelles (eau, biodiversité) sont réduits, voire même dépassés. La vigne qui nécessite moins de traitements du fait d'une large utilisation de variétés résistantes, est reconnue comme contribuant positivement à l'environnement. Elle permet également de maintenir le cadre paysager et culturel, qui intègre harmonieusement l'adoption de nouvelles technologies tout à fait respectueuses de l'environnement. Econome en eau, elle joue aussi un rôle de barrière aux incendies dont les risques sont accrus avec le changement climatique.

\section{Innovations de procédés et organisation de la filière pour un produit constant}

Le maintien de la viticulture dans les zones actuelles conforte une gouvernance représentative des acteurs traditionnels de la filière, mais aussi de la société civile du fait de la préoccupation écologique. Elle intègre de nouveaux experts viticulteurs, entrepreneurs écologiques et numériques, qui dominent la filière en y construisant et y apportant des connaissances scientifiques et professionnelles très pointues. La bonne organisation de la filière est favorable à son développement ; sa gouvernance est en mesure de fixer des objectifs de production clairs qui visent notamment à soutenir et intégrer les innovations à la vigne et à la cave. L'objectif de ces innovations est multiple. Il s'agit en amont de maintenir une différenciation par le terroir et les standards des qualités organoleptiques des vins, et, en aval, de favoriser la bonne perception de la consommation de vin dans l'opinion publique, en la rendant compatible avec 
Tableau 2.

\section{Chemin conduisant préférentiellement à une stratégie d'adaptation innovante}

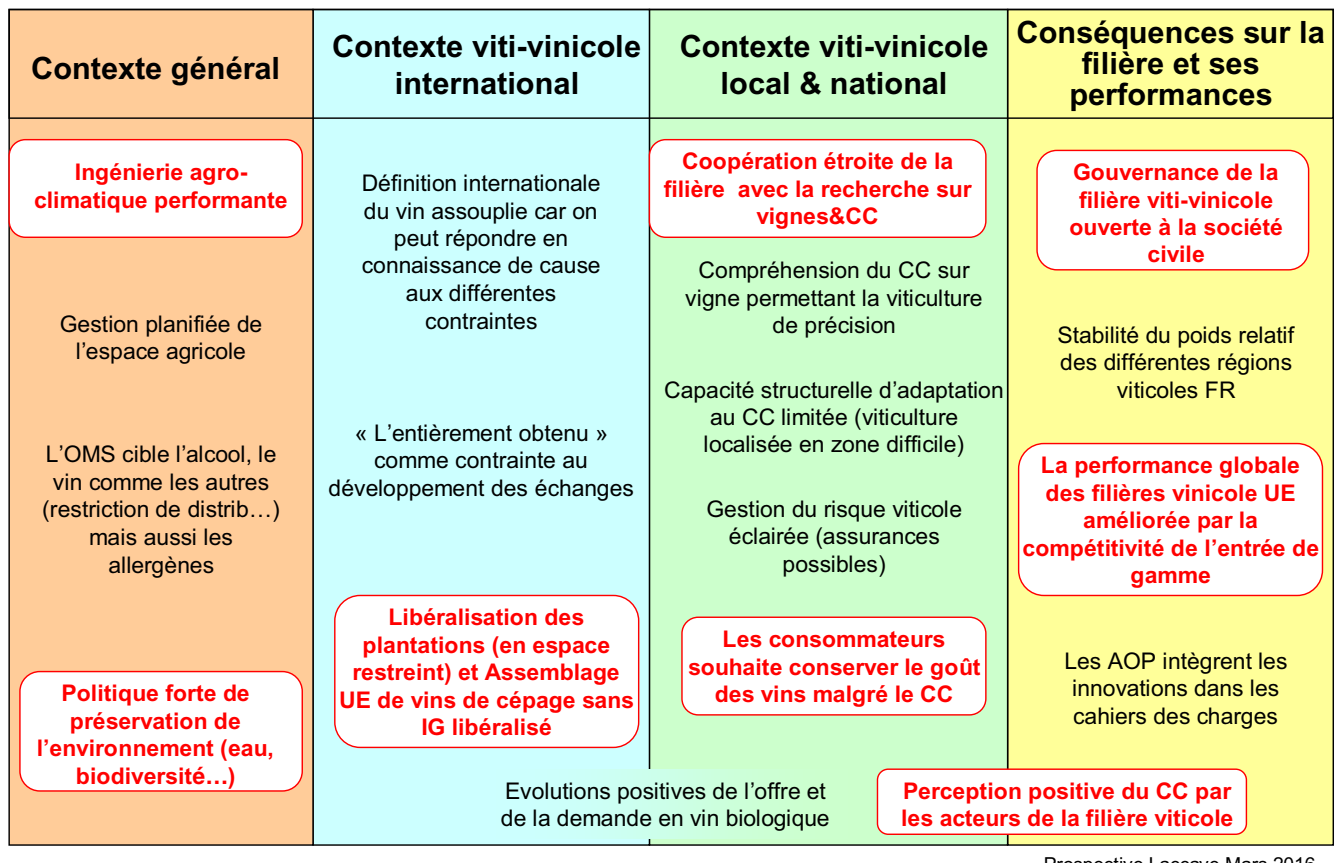

les préoccupations de santé et d'environnement. La R\&D, accompagnée par une politique de santé publique étatique efficace, vise notamment la réduction de la teneur des vins en alcool, en pesticides et en allergènes. Les AOC et IGP intègrent ces innovations - non sans certaines difficultés - dans leurs cahiers des charges. Profitant à la fois d'une connaissance accrue des mécanismes des liens au terroir, d'investissements en R\&D et d'assouplissements de la définition du vin, la différenciation par le terroir peut s'étendre à de nouvelles zones. Ainsi une nouvelle carte des terroirs viticoles se dessine à partir de la géographie actuelle, mais sans bouleversement majeur.

\section{Le chemin vers la stratégie nomade}

\section{Résumé \\ Dans un contexte marqué par une politique restrictive en matière d'alcool et une recherche focalisée sur la réduction des intrants, les consommateurs soucieux de retrouver le goût de l'origine de leurs vins, conduisent la viticulture, dépourvue des connaissances nécessaires permettant de maintenir même approximativement la constance qualitative espérée, à valoriser dans d'autres territoires la notoriété des grandes appellations et à descendre en plaine chercher l'eau.}

Cf. Tableau 3.

Le contexte : libéralisation sectorielle sous contrôle des attentes sociétales (alcool, résidus, ... )

Le contexte général est marqué par une poursuite de la mondialisation, une démographie mondiale croissante, et une pression globale dans les pays développés pour la prise en compte par l'agriculture de deux types d'attentes majeures : la santé et l'environnement (alcool, résidus de pesticides, ...)

Après la mise à l'index du tabac, l'activité de l'OMS se focalise sur l'alcool sans aller toutefois jusqu'à contraindre de manière significative les échanges et la distribution du vin, quelque peu «protégé » par son statut «agricole» (tout le contenu du vin demeure comme devant être issu du raisin). Néanmoins, les aspects négatifs (alcool, pesticides, ...) de la consommation de vin dominent les aspects positifs (culture, hédonisme, convivialité, ... ). La libéralisation se poursuit donc dans le secteur viti-vinicole sans remise en cause de la définition générale du vin.

Au plan international, les règles d'assemblage et de détermination de l'origine douanière non préférentielle du vin, ainsi que l'élargissement des pratiques œnologiques (notamment les pratiques de fractionnement et réassemblage des constituants du vin, mais aussi celles relatives à la diminution du degré alcoolique des vins) traduisent cette poursuite de la libéralisation sectorielle et permettent l'apparition d'assembleurs internationaux notamment près des nouveaux foyers de consommation.

Ces évolutions sont accompagnées dans l'UE d'une liberté totale de plantation, notamment pour résister à la concurrence internationale et faciliter une réimplantation rapide des vignobles, en particulier ceux dont l'activité est la plus handicapée par les effets du changement climatique.

Dans un tel contexte, et malgré les demandes des acteurs de la filière, l'Etat n'investit dans la R\&D vitivinicole que dans les domaines socialement acceptables tels que la réduction des intrants (y compris l'eau) et de la teneur en alcool des vins.

La collecte d'informations géo-localisées sur les sols viticoles et/ou sur les réponses physiologiques de la vigne face au changement climatique se révèle insuffisante 
Tableau 3

\section{Chemin conduisant préférentiellement à une stratégie d'adaptation nomade}

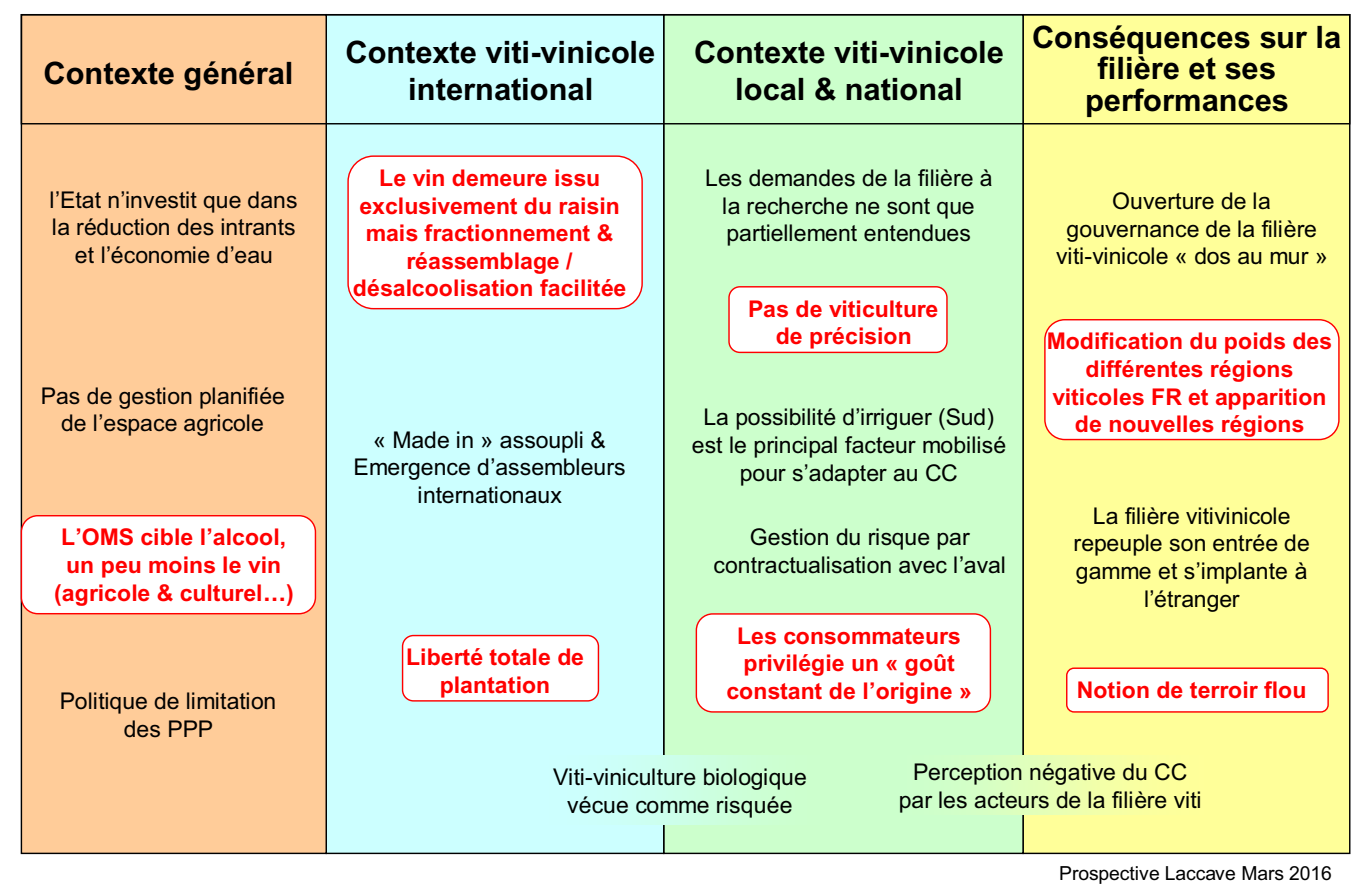

pour que la viticulture de précision puisse réellement se développer et que soient définis sur cette base de nouveaux itinéraires techniques permettant un maintien économiquement viable de l'exploitation et des pratiques viticoles socialement acceptables dans les zones les plus impactées par le changement climatique.

\section{La réaction des acteurs de la filière}

La notion de terroir demeure floue et ne diffuse pas dans le monde viti-vinicole et auprès des consommateurs. Les notoriétés historiquement acquises continuent de hiérarchiser les vins : origine, provenance, terroir, territoire, voire 《made by » ou signes de qualité à connotation géographique (type Ruby ou vin de glace) se confondent dans l'esprit d'une grande majorité de ménages acheteurs. L'intérêt des consommateurs pour la notion d'un « vin d'ici » réside dans la volonté de réduire les risques pris à l'achat, un produit de cette nature se devant de garantir une certaine constance organoleptique (sauf dans le segment haut de gamme pour des consommateurs avertis qui acceptent la variabilité). Ce « goût de l'origine », qui est en fait un goût constant et reconnaissable, conduit à ce que la typicité soit de plus en plus définie par des caractéristiques analysables.

La volonté de constance du profil gustatif global des produits dans un contexte de confidentialité de la viticulture de précision, conduit à chercher à obtenir ce résultat par une mobilité progressive du vignoble, qui s'accompagne de l'apparition de nouvelles combinaisons sol-climat-matériel végétal (notamment avec des cépages économes en intrants).

En effet, dans ce contexte de liberté de plantation, alors que les consommateurs attendent une certaine constance des produits, les solutions techniques pour adopter « sur place » les itinéraires de production aptes à satisfaire même approximativement cette attente ne sont pas disponibles. Dès lors :

- dans le sud, même si le fait de disposer dans son exploitation, ou dans son aire d'appellation d'origine protégée (AOP), d'une réserve foncière en altitude (fraicheur) devient un levier d'adaptation au changement climatique, on assiste majoritairement à une descente en plaine du vignoble méridional pour accéder à l'irrigation. Si le potentiel AOP en coteaux est menacé, les IGP et les vins de cépage sans IG peuvent voir alors leur potentiel croître ;

- dans le nord, la saturation des zones d'AOP notoires induisant un coût d'accès élevé à la production, ainsi que les restrictions croissantes à l'usage des pesticides, défavorisées par un contexte de changement, conduit les investisseurs (anciens ou nouveaux) à créer des vignobles dans les nouvelles marges climatiques du « nord » de l'UE. Ils cherchent à transporter en termes d'image la notoriété préalablement acquise de ces vignobles.

Ces évolutions ne favorisent pas la généralisation des pratiques de l'agriculture biologique, jugées généralement trop risquées pour répondre de manière rentable aux aléas climatiques. Face à l'augmentation de ces aléas, la gestion du risque par les viticulteurs passe par une contractualisation avec l'aval, combinée, selon les régions, avec des mécanismes classiques d'assurances ou de mutualisation.

Le changement climatique est majoritairement vécu comme une contrainte par la filière viti-vinicole. Sa gouvernance n'accepte d'ouvrir son périmètre historique que « dos au mur » : dans le sud, c'est l'utilisation significative de l'irrigation qui est le principal vecteur de l'ouverture de la gouvernance ; dans le nord c'est 
la volonté de répondre aux attentes sociétales en lien notamment avec l'usage des pesticides et de préserver l'image des produits.

Les poids relatifs des vignobles sont modifiés et la France viticole voit le modèle AOP, dont elle se sent dépositaire, fortement interrogé entre les notions de terroir et les attentes de constance relative des produits de la part des consommateurs, dans un contexte d'arsenal technique réduit. Globalement, la filière ne tire pas particulièrement bien son épingle du jeu au plan international, même si sa nouvelle pyramide productive lui permet de redevenir productrice d'entrée de gamme, et si, parmi les investisseurs des nouvelles marges viticoles nord-européennes, se trouvent des investisseurs français « originaires » de la filière.

\section{Le chemin vers la stratégie libérale}

Résumé
Dans un contexte plus libéral et assez favorable au
marché du vin, de nouveaux investisseurs, au niveau
de la production et surtout du négoce, conduisent à un
redéploiement de la viticulture entre des pôles irrigués,
quelques terroirs de notoriété ancienne et de nouveaux
vignobles bénéficiant du changement climatique. Des
vins personnalisés ou de marques régionales se
maintiennent, mais l'offre est majoritairement composée
de vins technologiques contrôlés par quelques firmes
d'aval. L'instabilité climatique, la concurrence entre
vignobles, la dérégulation et la domination de quelques
firmes finissent par fragiliser des entreprises viticoles
désorganisées qui ne peuvent bénéficier pleinement de la
R\&D. Cf Tableau 4.

\section{Un contexte plus libéral}

Dans un premier temps le secteur de la vigne et du vin poursuit son développement dans un contexte de mondialisation, d'intégration des économies européennes, d'alignement progressif des normes et règles des marchés du vin sur les produits agroalimentaires (primauté des marques, information du consommateur, ...), d'acceptation croissante de technologies maitrisées par des groupes privés, et de libéralisation des politiques agricole, économique et d'innovation.

En Europe, la gestion de l'espace agricole s'est assouplie, même si une planification se maintient localement selon les initiatives de collectivités (intercommunalités et régions). L'agriculture, comme la viticulture, sont 《débarrassées 》 de normes et de contrôles administratifs jugés trop contraignants, pour laisser une place plus importante aux mécanismes de marché, mettant davantage en jeu la responsabilité des entreprises et des consommateurs. Les questions de santé et d'environnement sont toujours plus présentes et l'image du vin finit par en être affectée. Le système des protections des IG s'est affaibli et a évolué selon les pays vers des systèmes de marques individuelles ou collectives.

\section{Un éclatement spatial}

Les plantations de vigne sont devenues libres, donnant lieu à une diversité de stratégies spatiales et de produits : i) des terres agricoles fertiles et irrigables (en plaine) sont plantées en vigne, utilisant largement la combinaison nouveaux cépages, irrigation et œnologie corrective pour limiter la variabilité climatique et s'adapter aux évolutions climatiques et de marché ;

ii) la viticulture est par contre en retrait dans de nombreux espaces difficiles et non irrigables (coteaux), y compris pour des zones d'appellation aujourd'hui reconnues. Quelques « spots» viticoles se maintiennent, soutenus par des investisseurs en quête d'authenticité (patrimoine bâti, nature) ou par des groupes de viticulteurs en résistance et ayant réussi à garder des prix élevés, en lien avec des stratégies œnotouristiques ;

iii) de nouvelles plantations de vignes se sont multipliées dans des zones aujourd'hui non viticoles (Bassin parisien, Bretagne, Normandie, Nord, espaces périurbains, ...) sans que toutefois de nouveaux vignobles importants et organisés ne s'affirment, du fait des risques climatiques et d'une concurrence sur le foncier.

Ainsi, la surface du vignoble s'est initialement maintenue puis s'est réduite par la suite. Mais elle s'est surtout éclatée, fragmentée avec une diversité de trajectoires régionales et une multitude de pôles en concurrence.

Une viticulture conquérante et rentable, puis fragilisée

La viticulture est restée globalement un secteur rentable sur une première partie de la période (une quinzaine d'années), soutenue par une croissance de la consommation à l'échelle mondiale et la valorisation de connaissances et d'innovations, permettant de gérer à moyen terme l'adaptation des vignes dans des contextes climatiques variés et pour différents types de vins (vignes hybrides puis OGM, résistantes aux maladies, à la sécheresse). Cette viticulture d'abord conquérante a été soutenue par de nouveaux investisseurs, au stade de la production, mais surtout du négoce. Le pouvoir de ce dernier s'est accru du fait de sa capacité à répondre (par son marketing) aux évolutions des consommateurs des métropoles (goût, santé), à contrôler l'élaboration du vin par des techniques œnologiques plus souples, mais aussi à gérer les risques climatiques par un approvisionnement diversifié et des stratégies d'assemblage, dans le cadre d'une contractualisation avec les producteurs des vignobles irrigués. Toutefois, la position renforcée de ces opérateurs d'aval, l'instabilité climatique croissante, les concurrences accrues entre «pôles viticoles » (anciens et nouveaux) et la dérégulation du secteur finissent par fragiliser une grande partie des entreprises viticoles françaises, dont l'organisation collective s'est affaiblie dans la plupart des régions.

Des vins sans complexe face àdes niches de vins de terroir ou «new art »

Quelques vins de terroirs se maintiennent soutenus par des logiques individuelles ou des stratégies « haut de gamme » de groupes continuant d'investir dans l'industrie du luxe, mais ils sont devenus marginaux. Des vins personnalisés jouant une différenciation 《 artistique » grâce à l'innovation et/ou de nouvelles localisations ont également 
Tableau 4

\section{Chemin conduisant préférentiellement à une stratégie d'adaptation libérale}

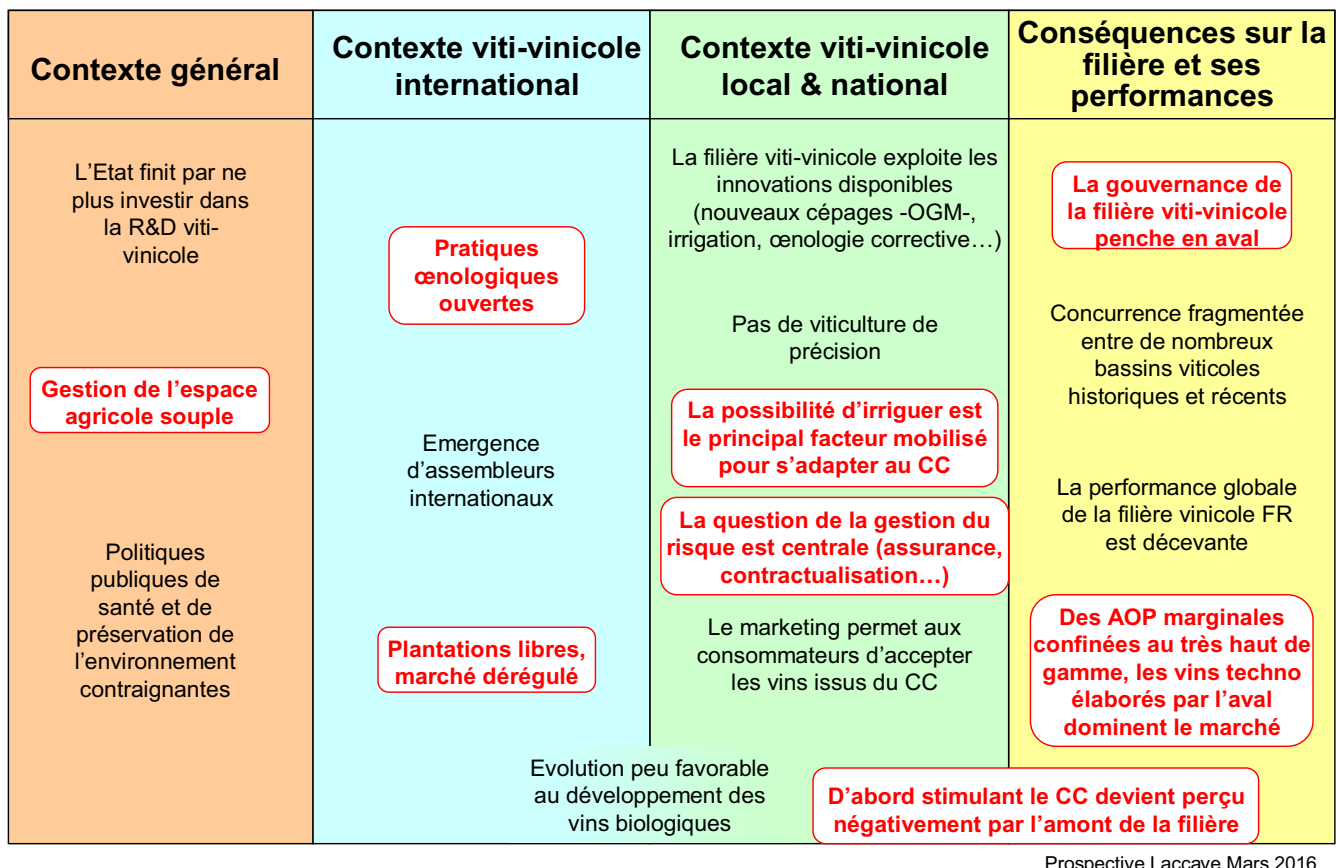

développé des marchés de niches, du local à l'international ( « new art wines », vins bretons ou des terrils, vin du quartier...). Ils réussissent même dans des conditions a priori difficiles du point de vue climatique. Mais l'essentiel de la production est constitué par des « vins sans complexes $\gg$, issus soit d'anciens vignobles d'appellation (IGP et AOP) reconstitués autour de pôles irrigués, soit de nouveaux vignobles implantés dans de nouvelles régions sans passé viticole. L'essentiel du marché du vin est constitué par des produits industrialisés, assemblés, voire aromatisés, visant à la fois :

i) le respect d'exigences environnementales et de santé,

ii) la limitation des impacts du changement climatique (plus que l'atténuation).

Des vins sous marque régionale ont aussi pu se maintenir, jouant sur des notoriétés historiques encore valorisantes (Champagne, Bordeaux, ...) et des accords avec certains négociants. Mais ces marques ne sont plus aussi fortes. La concurrence est vive au plan international et la France ne tire pas bénéfice de cette évolution de l'offre.

Des innovations technologiques discriminantes, ciblées et limitées face à la montée des risques

Dans un secteur fragilisé (après une période plutôt prospère), où l'amont est désorganisé et sans stratégie, et où l'image du vin est devenue plus négative, l'Etat n'investit plus en R\&D. Des innovations font donc défaut pour continuer à s'adapter au changement climatique dans tous les vignobles. La R\&D et l'accumulation d'informations géo-localisées sur les sols viticoles ou sur les réponses de la vigne au climat est contrôlée par des entreprises privées, donnant lieu à des innovations plus ciblées sur la génétique, l'irrigation et l'œnologie (corrective, aromatisante, ...). Ces investissements de $\mathrm{R} \& \mathrm{D}$ se révèlent à terme insuffisants pour que la viticulture de précision ne soit réellement utilisée à une large échelle (hors groupes privés) et pour qu'une nouvelle viticulture de terroir se développe en intégrant les enjeux de l'adaptation au changement climatique.

Face à la fragmentation et à la fragilisation des vignobles, à l'émergence de crises accentuées par le changement climatique, la gestion du risque devient un enjeu majeur, pouvant combiner contractualisation avec l'aval, recours aux assurances privées, intervention publique d'urgence et retrait ou diversification des vignobles.

\section{Conclusion}

La prospective est une «machine à raconter des histoires», pour avancer collectivement dans des réflexions les plus partagées et les plus interdisciplinaires possibles sur le devenir du système étudié, et pour aider à ce qu'émergent des stratégies d'actions partagées. La véracité ou la probabilité d'apparition des scénarios n'ont pas forcément d'intérêt... Ils sont évalués avant tout au regard de leur caractère attirant ou a contrario repoussoir, stimulant et appelant l'action dès maintenant ou a contrario appelant préparation et attention...

Les stratégies d'adaptation présentées ci-dessus et les chemins qui y mènent ne sont pas des images prédictives de l'avenir. Ce sont avant tout des outils pour stimuler la réflexion et la concertation des acteurs du secteur vitivinicole, en permettant notamment la prise en compte des interférences entre changement climatique et les autres évolutions possibles du contexte vitivinicole. 


\section{Références}

Quénol H. et al., 2014. Changement climatique et terroirs viticoles. Ed. Lavoisier, coll. Tech. \& Doc. 444 p.

Ollat, N., Touzard, J.-M. 2014. Long-term adaptation to climate change in viticulture and enology: Journal International des Sciences de la Vigne et du Vin, numéro spécial LACCAVE

Escudier J.L., Garcia de Cortazar I., Giraud-Héraud E., Le Roux, Ollat N., Quenol H., Touzard J.-M. (2016). Le vignoble français à l'épreuve du changement climatique, La Recherche, 513-514: 60-67, juillet-août 2016

Fuentes Espinoza, A., Giraud-Héraud, E., Pérès, S., Pons, A., Tempere, S. Darriet, Ph, (2016)., Réchauffement climatique et acceptabilité des vins par les consommateurs, Revue des Oenologues, 158, janvier 2016

Touzard J.-M., Ollat N. (2014). Stress hydrique et adaptation au changement climatique pour la viticulture et l'oenologie: le projet LACCAVE. Innovations Agronomiques, 38 (2014) : 131-140
Lebon E, Garcia De Cortazar Atauri I, 2014. Dans un contexte de changement climatique, quels sont les impacts de la sécheresse sur la vigne et sur le devenir des vignobles ? L'exemple du Languedoc. Innovations Agronomiques 38, 1-12

Ollat N, van Leeuwen C, Destrac Irvine A, Marguerit E, Duchêne E, Lebon E, Boursiquot JM, Torregrosa L, 2015. Changement climatique; quels seront les déterminants du choix du matériel végétal? Revue des Enologues 157S, 37-40

Sebillotte, M., Aigrain, P., Hannin, H., Sebillotte, C. 2003. Prospective : Vignes et Vins. Scénarios et défis pour la recherche et les acteurs. Bilan et Prospectives, Inra Editions

Aigrain, P., Hannin, H., Vert J. 2013 Perspectives d'évolution de la filière vignes et vins dans la région Languedoc-Roussillon à l'horizon 2025 CEP Analyses $\mathrm{n}^{\circ} 55$, Agreste

Aigrain, P., Brugière, F., Hannin, H., Touvron, Ch., 2014 Prospective ANIVIN de France. Les Etudes FranceAgriMer 